\title{
Multi-exciter stationary non-Gaussian random vibration test with time domain randomization
}

\author{
Ronghui Zheng ${ }^{\mathrm{a}, \mathrm{b}, *}$, Huaihai Chen ${ }^{\mathrm{a}}$, Dirk Vandepitte ${ }^{\mathrm{b}}$, Zhengbo Luo ${ }^{\mathrm{a}}$ \\ ${ }^{a}$ State Key Laboratory of Mechanics and Control of Mechanical Structures, College of Aerospace Engineering, Nanjing University of Aeronautics and \\ Astronautics, Nanjing 210016, China \\ ${ }^{\mathrm{b}}$ Department of Mechanical Engineering, KU Leuven, Kasteelpark Arenberg 41, Leuven 3001, Belgium
}

\section{A R T I C L E I N F O}

\section{Article history:}

Received 12 July 2018

Received in revised form 29 November 2018

Accepted 8 December 2018

\section{Keywords:}

MIMO vibration test

Random non-Gaussian vibrations

Kurtosis control

Skewness control

Time domain randomization

\begin{abstract}
A B S T R A C T
This paper presents a new control method for multi-input multi-output stationary nonGaussian random vibration test using time domain randomization. The control objectives are composed of response skewnesses, kurtoses and power spectral densities. The generation process of stationary and coupled reference non-Gaussian signals by specified reference skewnesses, kurtoses and spectra is analyzed. The reference non-Gaussian signals combined with system frequency response functions are then utilized to obtain the desired drive signals for dynamic inputs, in which the inverse system method in the frequency domain is employed. The primary advantages of the proposed methods are the high computational efficiency and simultaneous control of the time-frequency characteristics of response signals. In consideration of system cross coupling characteristics manifested in coherence and phase coefficients, the skewness and kurtosis tuning steps for each control channel are formulated by using a sequential phase modification method. The relationships between reference skewnesses, kurtoses and spectra are discussed and they reveal that the reference spectra have an influence on the settings of reference skewnesses and kurtoses, which implies that proper settings of reference skewnesses, kurtoses and spectra are necessary. A numerical example and a triaxial vibration test are provided and the results show the validity and feasibility of the proposed method.
\end{abstract}

(c) 2018 Elsevier Ltd. All rights reserved.

\section{Introduction}

Random vibration environmental testing is of considerable interest to engineers with great practical significance in many fields of science and engineering since several decades already. It is usually conducted as an effective means to assess the performance or durability of products under the specified Gaussian vibration environments in the laboratory or to reveal defects in product design [1-3]. However, many operational vibration environments in engineering structures are multidimensional non-Gaussian random, especially in the areas of aerospace, automotive and civil [4-6]. The fatigue life and failure modes of engineering structures are sometimes very sensitive to the exposed random vibration environments, two typical

\footnotetext{
* Corresponding author at: State Key Laboratory of Mechanics and Control of Mechanical Structures, College of Aerospace Engineering, Nanjing University of Aeronautics and Astronautics, Nanjing 210016, China.

E-mail addresses: rhzheng@nuaa.edu.cn (R. Zheng), chhnuaa@nuaa.edu.cn (H. Chen), dirk.vandepitte@kuleuven.be (D. Vandepitte), zbluo@nuaa.edu.cn (Z. Luo).
} 
examples are the comparisons of fatigue failure results of simultaneous and sequential axial vibrations and Gaussian and non-Gaussian vibrations [7-12].

The replication of simultaneous multidimensional non-Gaussian random vibration environments requires multi-exciter random vibration control. One method of multi-exciter testing is to use vibration shakers to excite the selected input points on the test article for the control of some monitoring points. The other common method uses vibration exciters on a rigid shaker table, with the test article rigidly connected to the shaker table by properly designed fixtures, and several acceleration sensors are mounted on the shaker table to control and monitor table vibrations induced by base excitation.

Classical multi-exciter random vibration control attempts to replicate the statistical properties, e.g. auto and cross power spectra of stationary Gaussian random vibration environments $[2,3,13,14]$. Smallwood suggested that the zero memory nonlinear transformation method can be introduced to obtain the desired non-Gaussian responses [15,16]. Zheng and Chen etc. proposed an inverse system method combined with sequential phase modification to allow for system cross coupling characteristics and to preserve spectral structures including multi-output response kurtoses, auto spectral densities and cross spectral densities which can be controlled simultaneously and independently $[17,18]$. However, the reference signals should be generated frame by frame successively which is computationally intensive and time consuming. Therefore, if there are many response channels to control or one channel reference kurtosis is large or skewness control is also considered, this method is limited because the elapsed time of generation of each frame reference signal increases with the number of control channels, values of control kurtoses and skewness control.

Another problem which should be tackled is the definition of reference skewnesses, kurtoses and spectra for multi-exciter stationary non-Gaussian random vibration control. The reference spectra are usually defined from processing measured data or artificial settings empirically. It just needs to guarantee the positive semi-definiteness of the reference spectral matrix to satisfy the physical realization. However, in the multi-exciter stationary non-Gaussian case, the definition of reference skewnesses and kurtoses should also be considered. It is obvious that reference skewnesses and kurtoses cannot be set arbitrarily due to the system cross coupling manifested as cross spectral densities, that is coherence and phase coefficients. For example, if the coherence coefficient in a two-input two-output reference spectral matrix equals one, meaning that two-channel random signals generated from reference spectral matrix originate from a single signal source, it is impossible to set the reference kurtoses of two channels arbitrarily. If the kurtosis of one channel is determined, the kurtosis of the other channel is also determined. Therefore, the definition of reference kurtoses and spectra before random vibration control test is necessary.

The time domain randomization technique proposed by Tebbs and Hunter has been widely employed in traditional random vibration control with high computational efficiency [19]. This paper provides a new closed loop control method for multi-exciter stationary non-Gaussian random vibration test with time domain randomization technique. The analyses and tuning steps of multi-output reference skewnesses and kurtoses by using sequential phase modification in the time domain randomization process are presented. The definition of multi-output reference skewnesses, kurtoses and spectra is also discussed. The primary advantages of the proposed control method lie in the efficient implementation manifested in the time domain randomization process and the simultaneous control of response skewnesses, kurtoses and power spectral densities. The rest of this paper is organized as follows. The proposed control method is formulated in Section 2. Subsequently, Section 3 discusses the definition of reference skewnesses, kurtoses and spectra as well as their relationships. A three-input three-output numerical example and its experimental results are illustrated in Sections 4 and 5 respectively. Finally, conclusions are given in Section 6.

\section{Non-Gaussian random vibration control method}

There are two families of multi-exciter testing methods which may be identified as square control and rectangular control according to the number of inputs and outputs. With square control the same number of inputs (drives) equals the number of outputs (controls) whereas rectangular control is characterized by different numbers of outputs. This paper focused on the general multi-exciter square control method.

Different from the traditional multi-exciter Gaussian case, the skewness and kurtosis describing the non-Gaussian features are considered in stationary non-Gaussian random vibration control. For an $n$ input $n$ output linear invariant dynamic system ( $n$ is a positive integer, $n \geq 2$ ), the control targets in time and frequency domains of matrix representation can be expressed as

$$
\begin{aligned}
& \mathbf{W}_{y}=\mathbf{W}_{\mathrm{Ref}} \\
& \mathbf{K}_{y}=\mathbf{K}_{\mathrm{Ref}} \\
& \mathbf{S}_{y y}=\mathbf{R}
\end{aligned}
$$

where $\mathbf{W}_{y}$ and $\mathbf{K}_{y}$ are the response skewness and kurtosis while $\mathbf{W}_{\text {Ref }}$ and $\mathbf{K}_{\text {Ref }}$ are the reference skewness and kurtosis respectively. $\mathbf{S}_{y y}$ is the response spectral density matrix. $\mathbf{R}$ is the specified positive semi-definite reference spectral density matrix and its off-diagonal elements can be written as 


$$
\begin{aligned}
& R_{j k}=\sqrt{\gamma_{j k}^{2} R_{j j} R_{k k}} e^{\mathrm{i} \alpha_{j k}}(j, k=1,2, \ldots, n ; j>k) \\
& R_{k j}=R_{j k}^{*}
\end{aligned}
$$

where diagonal elements $R_{j j}(j=1,2, \ldots, n)$ are the auto spectral densities and $\mathrm{i}$ is the imaginary unit. $\gamma_{j k}^{2}$ and $\alpha_{j k}$ are the coherence and phase coefficients between the $j$ th and $k$ th channels respectively. The superscript '*' represents the complex conjugate.

The crucial point of MIMO random vibration control is to generate time domain drive signals that can enforce the control channels to reproduce desired vibration responses within specified tolerances. According to the input-output relationships in linear time-invariant system, the first drive signals can be obtained in the frequency domain as

$$
\mathbf{D}=\mathbf{G}^{-1} \mathbf{C}
$$

where $\mathbf{G}$ is the system frequency response function matrix which is estimated before the vibration control test. $\mathbf{D}$ and $\mathbf{C}$ are the Fourier spectra of the drive signals and reference response signals respectively. The initial drive signals are only generated by the frequency response function matrix and reference spectral matrix, and later the response signals will be used to constitute a closed loop control algorithm to update drive signals. The flow chart of the proposed multi-exciter stationary non-Gaussian random vibration control method is shown in Fig. 1 and the detailed steps are given as follows.

First, the Cholesky decomposition is applied to the reference spectra $\mathbf{R}$ for the purpose of decoupling as

$$
\mathbf{R}=\mathbf{L L}^{\mathrm{H}}
$$

where superscript ' $H$ ' represents complex conjugate transpose and $\mathbf{L}$ is a lower triangular matrix. Then uniformly distributed random phase angles $\theta_{j}(j=1,2, \ldots, n)$ are supplemented for inverse Fast Fourier transform as

$$
\mathbf{u}_{m} \triangleq\left[\begin{array}{cccc}
\boldsymbol{F}^{-1}\left(l_{11} e^{\mathrm{i} \theta_{1}}\right) & 0 & 0 & 0 \\
\boldsymbol{F}^{-1}\left(l_{21} e^{\mathrm{i} \theta_{1}}\right) & \boldsymbol{F}^{-1}\left(l_{22} e^{\mathrm{i} \theta_{2}}\right) & 0 & 0 \\
\vdots & \vdots & \ddots & 0 \\
\boldsymbol{F}^{-1}\left(l_{n 1} e^{\mathrm{i} \theta_{1}}\right) & \boldsymbol{F}^{-1}\left(l_{n 2} e^{\mathrm{i} \theta_{2}}\right) & \ldots & \boldsymbol{F}^{-1}\left(l_{n n} e^{\mathrm{i} \theta_{n}}\right)
\end{array}\right]
$$

where $l_{j k}(j, k=1,2, \ldots, n ; j \geq k)$ are the elements of $\mathbf{L} . \boldsymbol{F}^{-1}$ denotes the inverse Fourier transform operation. To ensure the correlations between various channels, the row summation operation is performed to $\mathbf{u}_{m}$ and one frame random signal can be obtained as

$$
u_{j}=\left\{\begin{array}{lc}
\boldsymbol{F}^{-1}\left(l_{11} e^{\mathrm{i} \theta_{1}}\right) & j=1 \\
\underbrace{\sum_{k=1}^{j-1} \boldsymbol{F}^{-1}\left(l_{j k} e^{\mathrm{i} \theta_{k}}\right)}_{\triangleq u_{j, 1}}+\underbrace{\boldsymbol{F}^{-1}\left(l_{j j} e^{\mathrm{i} \theta_{j}}\right)}_{\triangleq u_{j, 2}} & j=2,3, \ldots, n
\end{array}\right.
$$

Eq. (8) shows that the elements in the same column are coupled due to the same random phase angles while elements in different columns are independent of each other due to the different and independent random phase angles.

Therefore, the skewness and kurtosis of row summed random signals in Eq. (9) can be calculated if the skewness and kurtosis of $u_{j, 1}$ and $u_{j, 2}$ are determined. In fact, the skewness $W_{x+y}$ of the summed two independent zero-mean random signals $x$ and $y$ can be calculated as

$$
W_{x+y}=\frac{\mathrm{E}\left[(x+y)^{3}\right]}{\mathrm{E}^{3 / 2}\left[(x+y)^{2}\right]}=\frac{\mathrm{E}\left[x^{3}+y^{3}+3 x^{2} y+3 x y^{2}\right]}{\mathrm{E}^{3 / 2}\left[x^{2}+y^{2}+2 x y\right]}=\frac{\mathrm{E}\left[x^{3}\right]+\mathrm{E}\left[y^{3}\right]}{\left(\mathrm{E}\left[x^{2}\right]+\mathrm{E}\left[y^{2}\right]\right)^{3 / 2}}=\frac{W_{x} \sigma_{x}^{3}+W_{y} \sigma_{y}^{3}}{\left(\sigma_{x}^{2}+\sigma_{y}^{2}\right)^{3 / 2}}
$$

Similarly, the kurtosis $K_{x+y}$ of the summed signal can be obtained as

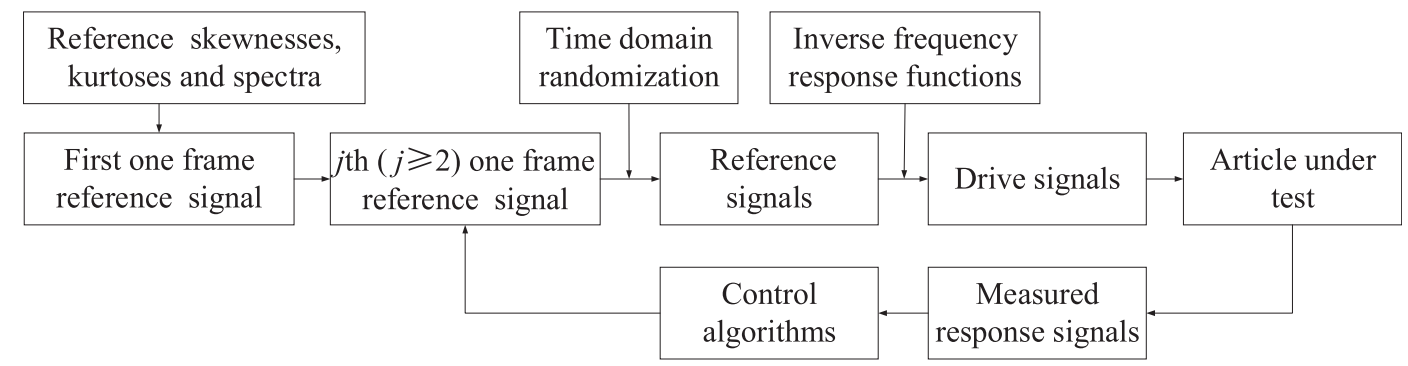

Fig. 1. Block diagram for closed loop control of stationary non-Gaussian random vibration test. 


$$
K_{x+y}=\frac{\mathrm{E}\left[(x+y)^{4}\right]}{\mathrm{E}^{2}\left[(x+y)^{2}\right]}=\frac{K_{x} \sigma_{x}^{4}+K_{y} \sigma_{y}^{4}+6 \sigma_{x}^{2} \sigma_{y}^{2}}{\left(\sigma_{x}^{2}+\sigma_{y}^{2}\right)^{2}}
$$

where E[.] represents the mathematical expectation operation. $W_{x}$ and $W_{y}$ are the skewnesses of $x$ and $y$ while $K_{x}$ and $K_{y}$ are the kurtoses of $x$ and $y$ respectively. $\sigma_{x}$ and $\sigma_{y}$ are the standard deviations of $x$ and $y$ respectively.

Based on the above analysis, one frame reference non-Gaussian signal can be generated from reference skewnesses, kurtoses and spectra by modifying the random phase angles in sequence. The tuning process is realized by three successive steps:

Step 1 . Modify random phase angles $\theta_{1}$ such that the signal from channel one $u_{1}$ satisfies its reference skewness and kurtosis. Here, the improved zero memory nonlinear transformation method is used to modify phase angles [19]. The skewness and kurtosis of the first column in Eq. (8) change because the random phase angles $\theta_{1}$ are modified.

Step 2. Modify random phase angles $\theta_{2}$ such that the signal from channel two $u_{2}$ satisfies its reference skewness and kurtosis. Note that $u_{2}$ is composed of $u_{2,1}$ and $u_{2,2}$, and the skewness and kurtosis of $u_{2}$ can be tuned only by tuning the skewness and kurtosis of $u_{2,2}$ because $u_{2,1}$ is determined due to the modified $\theta_{1}$.

Step 3. Modify random phase angles $\theta_{3}-\theta_{n}$ sequentially such that all corresponding channel signals satisfy their reference skewnesses and kurtoses. The phase modification process must be in sequence to avoid problems of cross coupling.

Therefore, only the skewness and kurtosis of $u_{j, 2}$ can be adjusted to tune skewness and kurtosis of channel $j(j=2,3, \ldots, n)$. According to Eqs. ((10) and (11)), the skewness and kurtosis of $u_{j, 2}$ that need to be adjusted can be expressed as

$$
\begin{aligned}
& W_{j, 2}=\left(\lambda_{j}^{2}+1\right)^{3 / 2} W_{j}-\lambda_{j}^{3} W_{j, 1} \\
& K_{j, 2}=\left(\lambda_{j}^{2}+1\right)^{2} K_{j}-\lambda_{j}^{4} K_{j, 1}-6 \lambda_{j}^{2}
\end{aligned}
$$

where

$$
\lambda_{j}=\frac{\sigma_{j, 1}}{\sigma_{j, 2}}(j=2,3, \ldots n)
$$

$W_{j, 1}$ and $W_{j, 2}$ are the skewnesses of $u_{j, 1}$ and $u_{j, 2}$ and $K_{j, 1}$ and $K_{j, 2}$ are the kurtoses of $u_{j, 1}$ and $u_{j, 2}$, respectively. $\sigma_{j, 1}$ and $\sigma_{j, 2}$ are the standard deviations of $u_{j, 1}$ and $u_{j, 2}$ respectively. Note that all random signals in this paper are zero mean. $W_{j}$ and $K_{j}$ are $j$ th reference skewness and kurtosis respectively.

Afterwards, a time domain randomization technique is used in place of generating a new one frame random signal to produce the desired stationary reference non-Gaussian signals. Because of the high computational efficiency of the time domain randomization technique, the realization of stationary time domain reference non-Gaussian signals can be easily achieved. The analysis of the time domain randomization process by one frame reference non-Gaussian signal follows three substeps:

Step a. Pick different points randomly and synchronously for each column random signal in Eq. (8) and perform a circular shift to the generated one frame random signal to obtain a reconstructed random signal. This operation does not change the skewness, kurtosis and power spectral density because the time domain amplitudes of signals remain unchanged. Step $b$. Repeat Step a several times to generate many frame random signals that are windowed and overlapped to compose stationary random signals. The operation of windowing and overlapping decreases the skewness and kurtosis of a random signal; the modified skewness and kurtosis are related to the window function and overlap factor. In this paper, a halfsine window with an overlap factor of 2 is used. The skewness and kurtosis relationships before and after the operation of windowing and overlapping are thus given as

$$
\begin{aligned}
& K_{a}=\frac{3}{4} K_{b}+\frac{3}{4} \\
& W_{a}=\frac{8}{3 \pi} W_{b}
\end{aligned}
$$

where $K_{a}$ and $W_{a}$ are the skewness and kurtosis after windowing and overlapping while $K_{b}$ and $W_{b}$ are the skewness and kurtosis before windowing and overlapping. Eqs. ((12) and (13)) are modified to

$$
\begin{aligned}
& W_{j, 2}=\frac{3 \pi}{8}\left(\lambda_{j}^{2}+1\right)^{3 / 2} W_{j}-\lambda_{j}^{3} W_{j, 1} \\
& K_{j, 2}=\frac{4}{3}\left(\lambda_{j}^{2}+1\right)^{2} K_{j}-\lambda_{j}^{4} K_{j, 1}-\lambda_{j}^{4}-8 \lambda_{j}^{2}-1
\end{aligned}
$$


Step c. Sum the random signals in each row to obtain coupled reference non-Gaussian signals as shown in Eq. (9).

In a final step, the Fourier transform is applied to the generated time domain reference non-Gaussian signals to obtain Fourier spectra $\mathbf{C}$ in Eq. (6), the desired drive signals are then obtained by an inverse Fourier transform of $\mathbf{D}$. The drive signals are updated according to the deviations between the response and reference signals and the control algorithm is as follows:

$$
\begin{aligned}
& \boldsymbol{L}_{\text {new }}=\left(\boldsymbol{L}_{y}^{-1}\right)^{\eta_{L}} \boldsymbol{L}_{\text {old }} \\
& \boldsymbol{W}_{\text {new }}=\eta_{S}\left(\boldsymbol{W}_{\text {Ref }}-\boldsymbol{W}_{y}\right) \boldsymbol{W}_{\text {old }} \\
& \boldsymbol{K}_{\text {new }}=\left(\boldsymbol{K}_{\text {Ref }} \boldsymbol{K}_{y}^{-1}\right)^{\eta_{K}} \boldsymbol{K}_{\text {old }}
\end{aligned}
$$

where $\mathbf{L}_{\text {new }}$ is used to update $\mathbf{L}$ in Eq. (7) and $\mathbf{L}_{y}$ represents the Cholesky decomposition of the present response spectra $\boldsymbol{S}_{y y}$. $\mathbf{W}_{\text {new }}$ and $\mathbf{K}_{\text {new }}$ are used to update the skewness and kurtosis in Eqs. ((17) and (18)) respectively. $\mathbf{W}_{y}$ and $\mathbf{K}_{y}$ are the present response skewness and kurtosis respectively. $\eta_{L}, \eta_{S}$ and $\eta_{K}$ are spectral, skewness and kurtosis convergence coefficients respectively.

\section{Discussion}

This section discusses the definition of reference skewnesses, kurtoses and spectra in MIMO stationary non-Gaussian random vibration control. The influence of $\lambda_{j}$ on the skewness and kurtosis tuning process is explained first, and then the definition of reference skewnesses, kurtoses and spectra is analyzed.

According to Eqs. ((17) and (18)), jth reference skewness and kurtosis can be expressed as

$$
\begin{aligned}
& W_{j}=\frac{8}{3 \pi} \frac{\lambda_{j}^{3}}{\left(\lambda_{j}^{2}+1\right)^{3 / 2}} W_{j, 1}+\underbrace{\frac{8}{3 \pi} \frac{1}{\left(\lambda_{j}^{2}+1\right)^{3 / 2}}}_{\triangleq W_{\text {Amp. } j}} W_{j, 2} \\
& K_{j}=\frac{3}{4} \frac{\lambda_{j}^{4}}{\left(\lambda_{j}^{2}+1\right)^{2}} K_{j, 1}+\frac{3}{4} \frac{\lambda_{j}^{4}+8 \lambda_{j}^{2}+1}{\left(\lambda_{j}^{2}+1\right)^{2}}+\underbrace{\frac{3}{4} \frac{1}{\left(\lambda_{j}^{2}+1\right)^{2}}}_{\triangleq K_{\text {Amp } . j}} K_{j, 2}
\end{aligned}
$$

Eq. (22) shows that the $W_{j}$ depends on $W_{j, 1}$ and $W_{j, 2}$, and each of them contributes to some extent to the skewness modification. $W_{j, 1}$ is determined in $j$ th channel tuning process because of previously modified random angles, and thus only $W_{j, 2}$ can be used to tune the channel skewness. In theory, the tuning process of skewness and kurtosis has nothing to do with the value of $\lambda_{j}$. However, if $\lambda_{j}$ is much larger than 1 , the skewness amplification coefficient $W_{\mathrm{Amp}, j}$ is very small so that the contribution of $W_{j, 2}$ to $W_{j}$ is very small because of the small ratio. In other words, no matter how $W_{j, 2}$ is tuned, the value of $W_{j}$ changes very slowly. As a result, it is possible that $W_{j, 2}$ exceeds the maximum range of $u_{j, 2}$ that can be tuned. Furthermore, the larger the value of $W_{j, 2}$, the longer it takes to complete the tuning process. It is similar to the kurtosis tuning process in

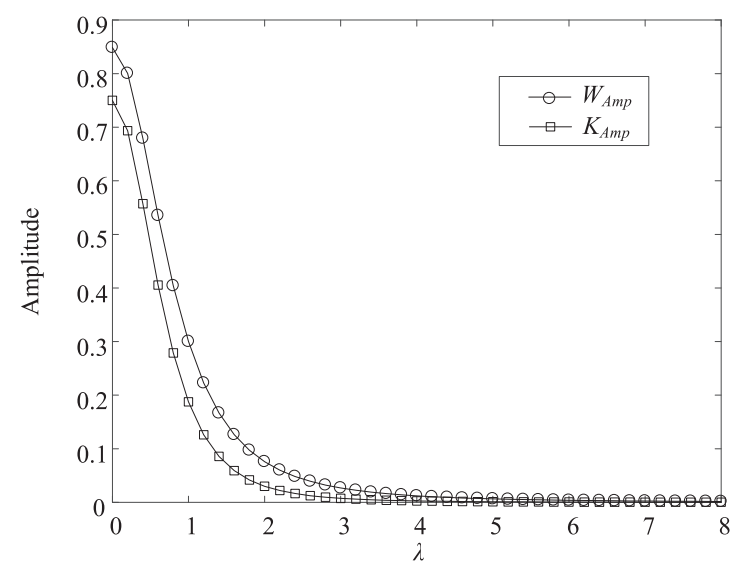

Fig. 2. Different values of amplification coefficients $W_{\mathrm{Amp}}$ and $K_{\mathrm{Amp}}$ with $\lambda$. 
Eq. (23) that $\lambda_{j}$ should not be very large. Fig. 2 shows the amplification coefficients $W_{\text {Amp }}$ and $K_{\text {Amp }}$ changes with $\lambda$ in the range $[0,8]$. If $\lambda=0$, that means that each channel is independent of each other and that coupling problem do not occur, the tuned $W_{j, 2}$ and $K_{j, 2}$ have full contributions on $W_{j}$ and $K_{j}$ modifications, respectively. If $\lambda_{j} \geq 2$, the contributions of tuned $W_{j, 2}$ and $K_{j, 2}$ are limited. Therefore, the value of $\lambda_{j}$ is very important for the skewness and kurtosis tuning processes.

It is also noteworthy that the reference kurtosis of the $j$ th channel cannot be smaller than $K_{j}^{\min }$ which is expressed as

$$
K_{j}^{\min }=\frac{3}{4} \frac{\lambda_{j}^{4}}{\left(\lambda_{j}^{2}+1\right)^{2}} K_{j, 1}+\frac{3}{4} \frac{\lambda_{j}^{4}+8 \lambda_{j}^{2}+4}{\left(\lambda_{j}^{2}+1\right)^{2}}
$$

The kurtosis tuning process is applied for a non-Gaussian random signal with kurtosis greater than 3 in this paper. The setting of reference skewness for each channel does not have this limitation because skewness can be a positive or negative value. However, the reference skewness and kurtosis should be set properly. In addition to limitations due to minimum or maximum skewness and kurtosis of each tuned random signal also calculation time and efficiency should be taken into account.

The above discussion shows that it is very important to determine the most appropriate value $\lambda_{j}$ for the skewness and kurtosis tuning processes. Eq. (14) shows that the $\lambda_{j}$ value is related with the reference spectra. To better understand the relationship between $\lambda_{j}$ and reference spectra, let $n$ be equal to 3 . Therefore, the reference spectral matrix $\mathbf{R}$ and lower triangular matrix $\mathbf{L}$ can be written as

$$
\begin{aligned}
& \boldsymbol{R}=\left[\begin{array}{ccc}
R_{11} & \gamma_{12} \sqrt{R_{11} R_{22}} e^{-i \alpha_{12}} & \gamma_{13} \sqrt{R_{11} R_{33}} e^{-i \alpha_{13}} \\
\gamma_{12} \sqrt{R_{11} R_{22}} e^{i \alpha_{12}} & R_{22} & \gamma_{23} \sqrt{R_{22} R_{33}} e^{-i \alpha_{23}} \\
\gamma_{13} \sqrt{R_{11} R_{33}} e^{i \alpha_{13}} & \gamma_{23} \sqrt{R_{22} R_{33}} e^{i \alpha_{23}} & R_{33}
\end{array}\right] \\
& \mathbf{L}=\left[\begin{array}{ccc}
\sqrt{R_{11}} & 0 & 0 \\
\gamma_{12} \sqrt{R_{22}} e^{i \alpha_{12}} & \sqrt{1-\gamma_{12}^{2}} \sqrt{R_{22}} & 0 \\
\gamma_{13} \sqrt{R_{33}} e^{i \alpha_{13}} & l_{32} & l_{33}
\end{array}\right]
\end{aligned}
$$

where

$$
\begin{gathered}
l_{32}=\frac{\sqrt{\gamma_{12}^{2} \gamma_{13}^{2}+\gamma_{23}^{2}-2 \gamma_{12} \gamma_{13} \gamma_{23} \cos \left(\alpha_{12}+\alpha_{23}-\alpha_{13}\right)}}{\sqrt{1-\gamma_{12}^{2}}} \sqrt{R_{33}} e^{i \phi_{23}} \\
\tan \phi_{23}=\frac{\gamma_{23} \sin \alpha_{23}+\gamma_{12} \gamma_{13} \sin \left(\alpha_{12}-\alpha_{13}\right)}{\gamma_{23} \cos \alpha_{23}-\gamma_{12} \gamma_{13} \sin \left(\alpha_{12}-\alpha_{13}\right)} \\
l_{33}=\frac{\sqrt{1-\gamma_{12}^{2}-\gamma_{13}^{2}-\gamma_{23}^{2}+2 \gamma_{12} \gamma_{13} \gamma_{23} \cos \left(\alpha_{12}+\alpha_{23}-\alpha_{13}\right)}}{\sqrt{1-\gamma_{12}^{2}}} \sqrt{R_{33}}
\end{gathered}
$$

According to Eq. (14), $\lambda_{j}$ can be obtained as

$$
\begin{gathered}
\lambda_{2}=\frac{\sigma_{2,1}}{\sigma_{2,2}}=\frac{\int_{\omega_{1}}^{\omega_{2}} \gamma_{12} \sqrt{R_{22}} \mathrm{~d} \omega}{\int_{\omega_{1}}^{\omega_{2}} \sqrt{1-\gamma_{12}^{2}} \sqrt{R_{22}} \mathrm{~d} \omega} \\
\lambda_{3}=\frac{\sigma_{3,1}}{\sigma_{3,2}}=\frac{\int_{\omega_{1}}^{\omega_{2}} \frac{\sqrt{\gamma_{13}^{2}+\gamma_{23}^{2}-2 \gamma_{12} \gamma_{13} \gamma_{23} \cos \left(\alpha_{12}+\alpha_{23}-\alpha_{13}\right)}}{\sqrt{1-\gamma_{12}^{2}}} \sqrt{R_{33}} \mathrm{~d} \omega}{\int_{\omega_{1}}^{\omega_{2}} \frac{\sqrt{1-\gamma_{12}^{2}-\gamma_{13}^{2}-\gamma_{23}^{2}+2 \gamma_{12} \gamma_{13} \gamma_{23} \cos \left(\alpha_{12}+\alpha_{23}-\alpha_{13}\right)}}{\sqrt{1-\gamma_{12}^{2}}} \sqrt{R_{33}} \mathrm{~d} \omega}
\end{gathered}
$$

For two-input two-output cases, $\lambda_{2}$ is only determined by reference auto spectral densities and by the coherence coefficient. If the coherence and phase coefficients are constant at each spectral line, Eq. (29) can be simplified to

$$
\begin{gathered}
\lambda_{2}=\frac{\gamma_{12}}{\sqrt{1-\gamma_{12}^{2}}} \\
\lambda_{3}=\frac{\sqrt{\gamma_{13}^{2}+\gamma_{23}^{2}-2 \gamma_{12} \gamma_{13} \gamma_{23} \cos \left(\alpha_{12}+\alpha_{23}-\alpha_{13}\right)}}{\sqrt{1-\gamma_{12}^{2}-\gamma_{13}^{2}-\gamma_{23}^{2}+2 \gamma_{12} \gamma_{13} \gamma_{23} \cos \left(\alpha_{12}+\alpha_{23}-\alpha_{13}\right)}}
\end{gathered}
$$


In this case, $\lambda_{j}$ values are only related with coherence and phase coefficients. If $\gamma_{12}$ is close to one, the value of $\lambda_{2}$ is very large which makes the contributions of $W_{j, 2}$ and $K_{j, 2}$ to its corresponding channel skewness and kurtosis very small.

Therefore, with regard to the settings of coherence and phase coefficients, not only the positive semi-definite of the reference spectral matrix should be guaranteed, but also the feasibility of skewness and kurtosis tuning processes should be considered. A pre-test procedure for checking the values of $\lambda_{j}$ can be done to ensure that the random vibration control test can be carried out successfully.

\section{Numerical example}

This section illustrates the proposed method on a system with an Aluminum cantilever beam. The validation study is conducted purely through simulation, but model parameters are set to represent a simple physical realization. The physical parameters of the beam and the locations of the excitation and control points are shown in Fig. 3. The modal damping ratios are set to 0.03 , the elastic modulus is $72 \mathrm{GPa}$ and the mass density is $2700 \mathrm{~kg} / \mathrm{m}^{3}$. The plots of frequency response functions of the system are shown in Fig. 4.

The reference spectra of three control points are set in Tables 1 and 2 . Only values of the power spectral densities at the break points are given and values at other frequency points are calculated by linear interpolation on a logarithmic scale. The control frequency band for simulation is set from $20 \mathrm{~Hz}$ to $2000 \mathrm{~Hz}$ with 800 spectral lines. The reference skewnesses and

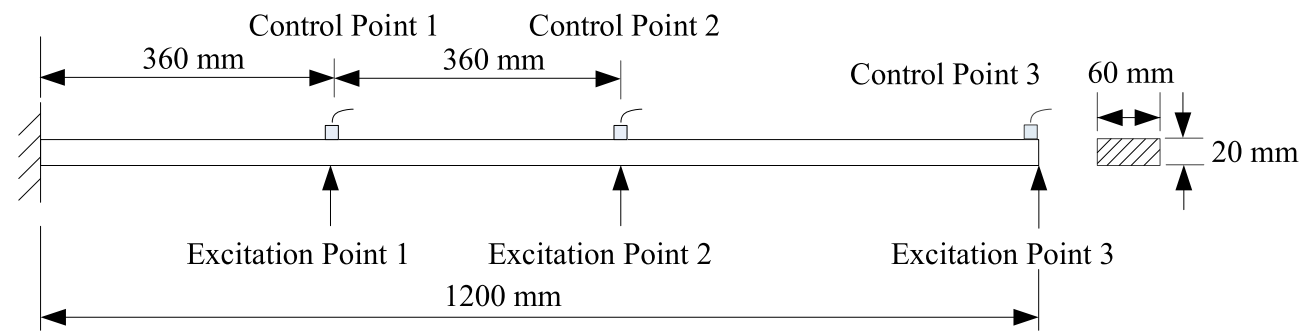

Fig. 3. Cantilever beam for simulation test.
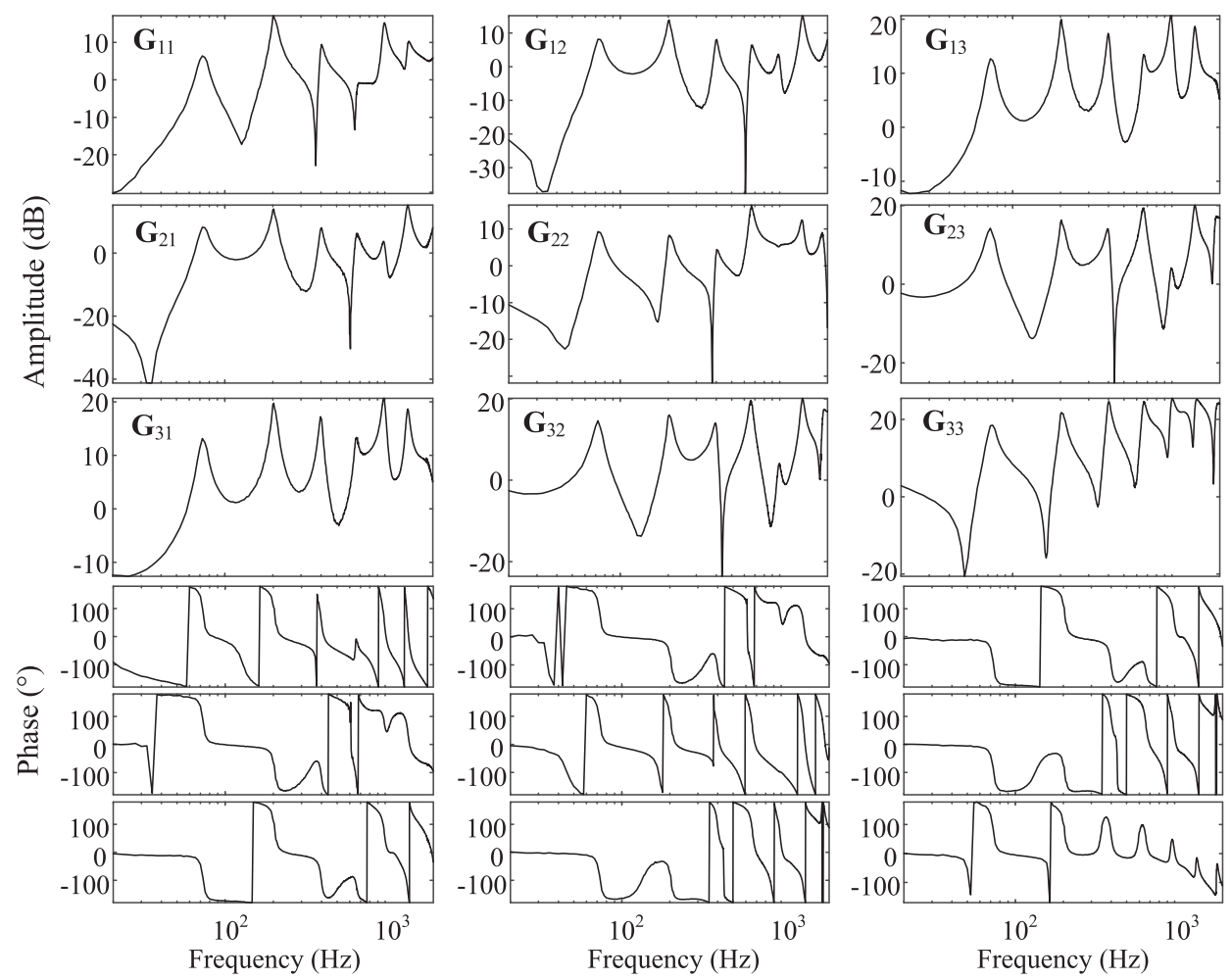

Fig. 4. Plots of frequency response functions of simulation system. 
Table 1

Reference auto spectral densities for simulation test.

\begin{tabular}{llll}
\hline Break point $(\mathrm{Hz})$ & $\mathrm{R}_{11}\left(\mathrm{~g}^{2} / \mathrm{Hz}\right)$ & $\mathrm{R}_{22}\left(\mathrm{~g}^{2} / \mathrm{Hz}\right)$ & $\mathrm{R}_{33}\left(\mathrm{~g}^{2} / \mathrm{Hz}\right)$ \\
\hline 20 & $1 \mathrm{e}-5$ & $1 \mathrm{e}-5$ & $1 \mathrm{e}-5$ \\
100 & $1 \mathrm{e}-4$ & $1 \mathrm{e}-4$ & $1 \mathrm{e}-4$ \\
1000 & $1 \mathrm{e}-4$ & $1 \mathrm{e}-4$ & $1 \mathrm{e}-4$ \\
2000 & $1 \mathrm{e}-5$ & $1 \mathrm{e}-5$ & $1 \mathrm{e}-4$ \\
\hline
\end{tabular}

Table 2

Reference cross spectral densities for simulation test.

\begin{tabular}{llllll}
\hline Break point $(\mathrm{Hz})$ & Coherence $\gamma_{12}^{2}$ & Coherence $\gamma_{13}^{2}$ & Coherence $\gamma_{23}^{2}$ & Phase $\alpha_{12}\left(^{\circ}\right)$ & Phase $\alpha_{13}\left({ }^{\circ}\right)$ \\
\hline 20 & 0.7 & 0 & 0.1 & 45 & - \\
100 & 0.7 & 0 & 0.1 & 45 & -60 \\
1000 & 0.7 & 0 & 0.1 & 45 & -60 \\
2000 & 0.7 & 0 & 0.1 & 45 & -60 \\
\hline
\end{tabular}

Table 3

Reference skewnesses and kurtoses for simulation test.

\begin{tabular}{lll}
\hline Control point & Skewness & Kurtosis \\
\hline 1 & 0.3 & 6 \\
2 & 0.2 & 6 \\
3 & 0 & 8 \\
\hline
\end{tabular}
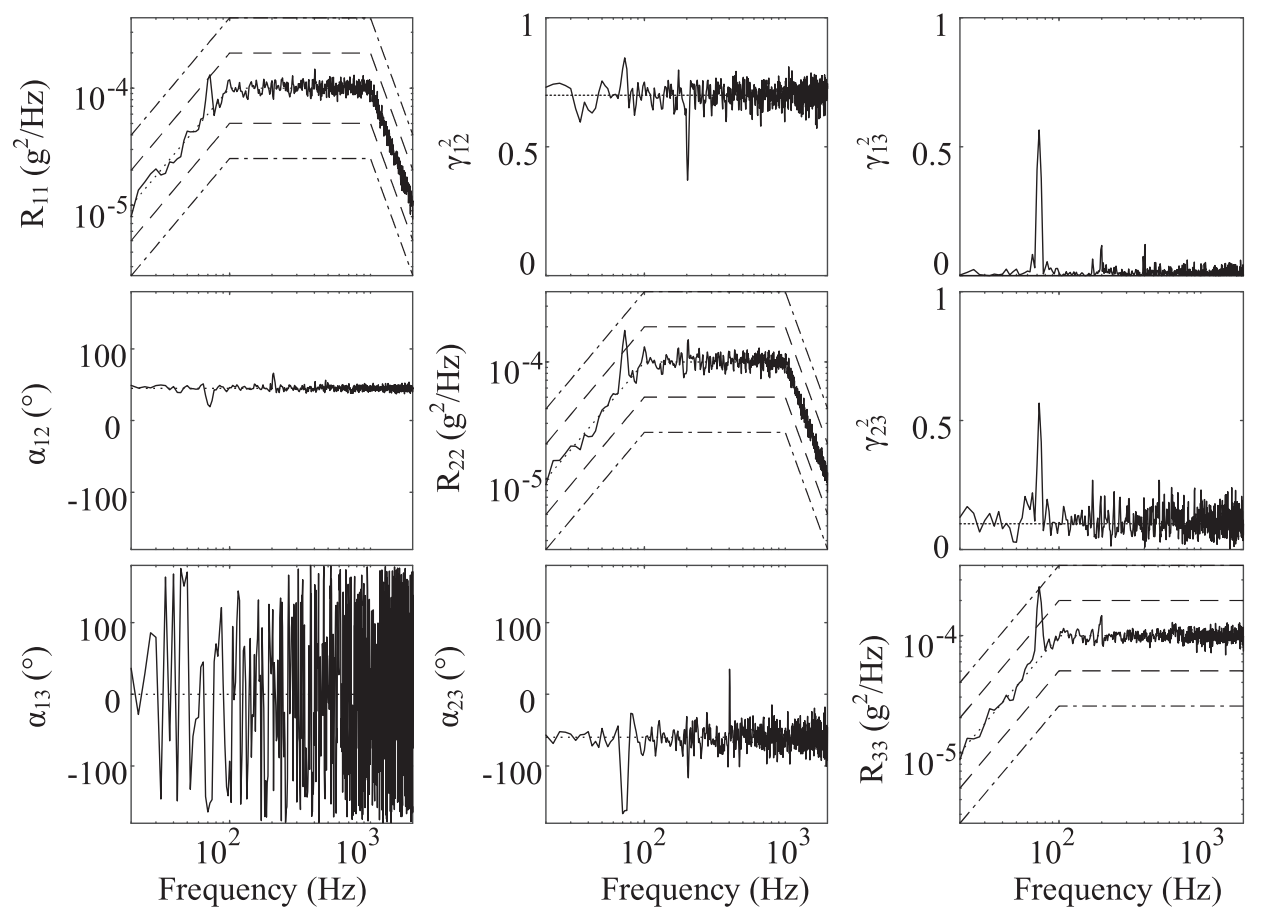

$$
\begin{array}{ll} 
--- \pm 3 \mathrm{~dB} \text { alarm limits } & \text { Reference spectrum } \\
--- \pm 6 \mathrm{~dB} \text { abort limits } & \text { Response spectrum }
\end{array}
$$

Fig. 5. Uncontrolled response spectra of three control points.

kurtoses of three control points are set in Table 3. The tolerances for the auto-spectral densities are set to $\pm 3 \mathrm{~dB}$ alarm limits and $\pm 6 \mathrm{~dB}$ as abort limits. The tolerances for skewnesses, kurtoses and the tolerances for cross spectral densities are not set in this example. 

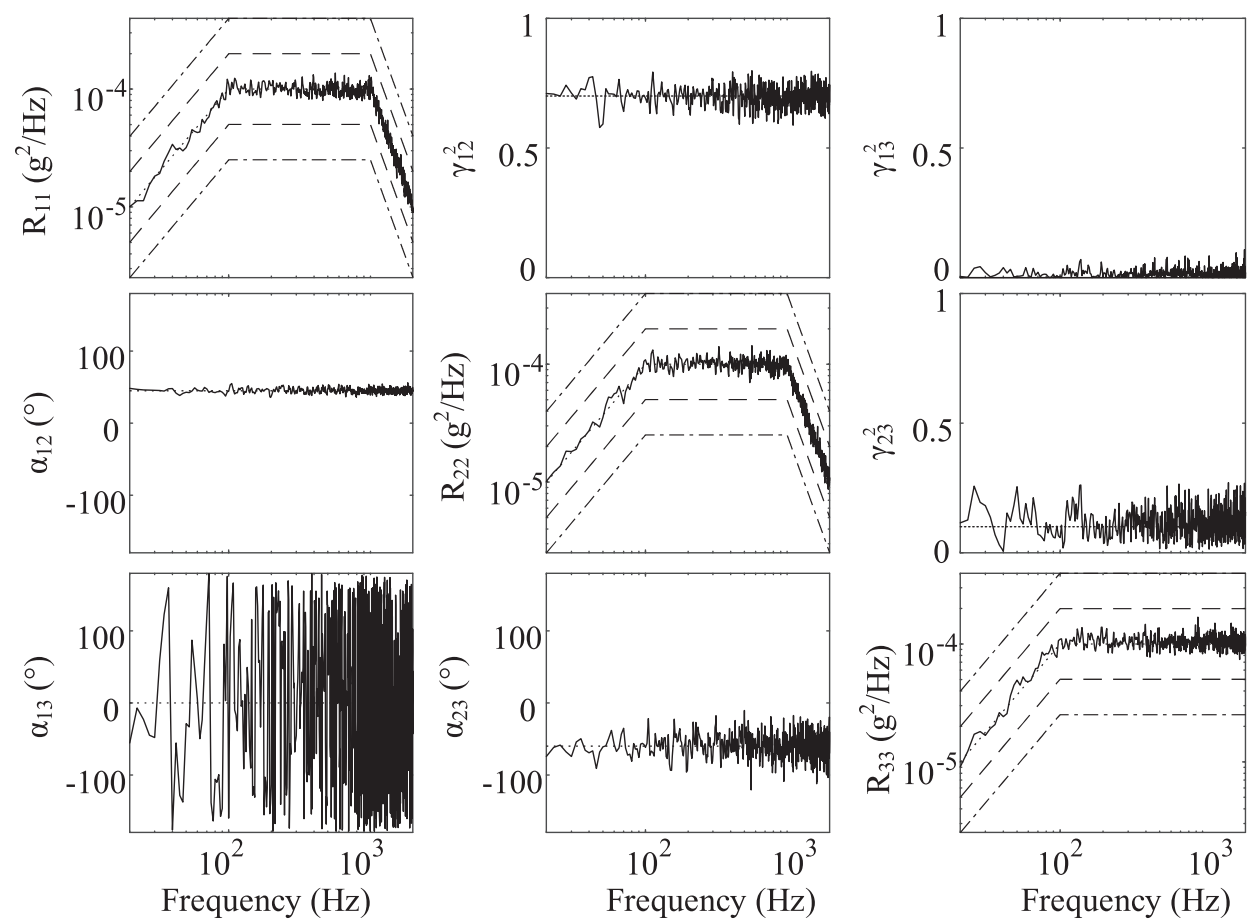

$--- \pm 3 \mathrm{~dB}$ alarm limits
$--- \pm 6 \mathrm{~dB}$ abort limits

Reference spectrum

Response spectrum

Fig. 6. Controlled response spectra of three control points.
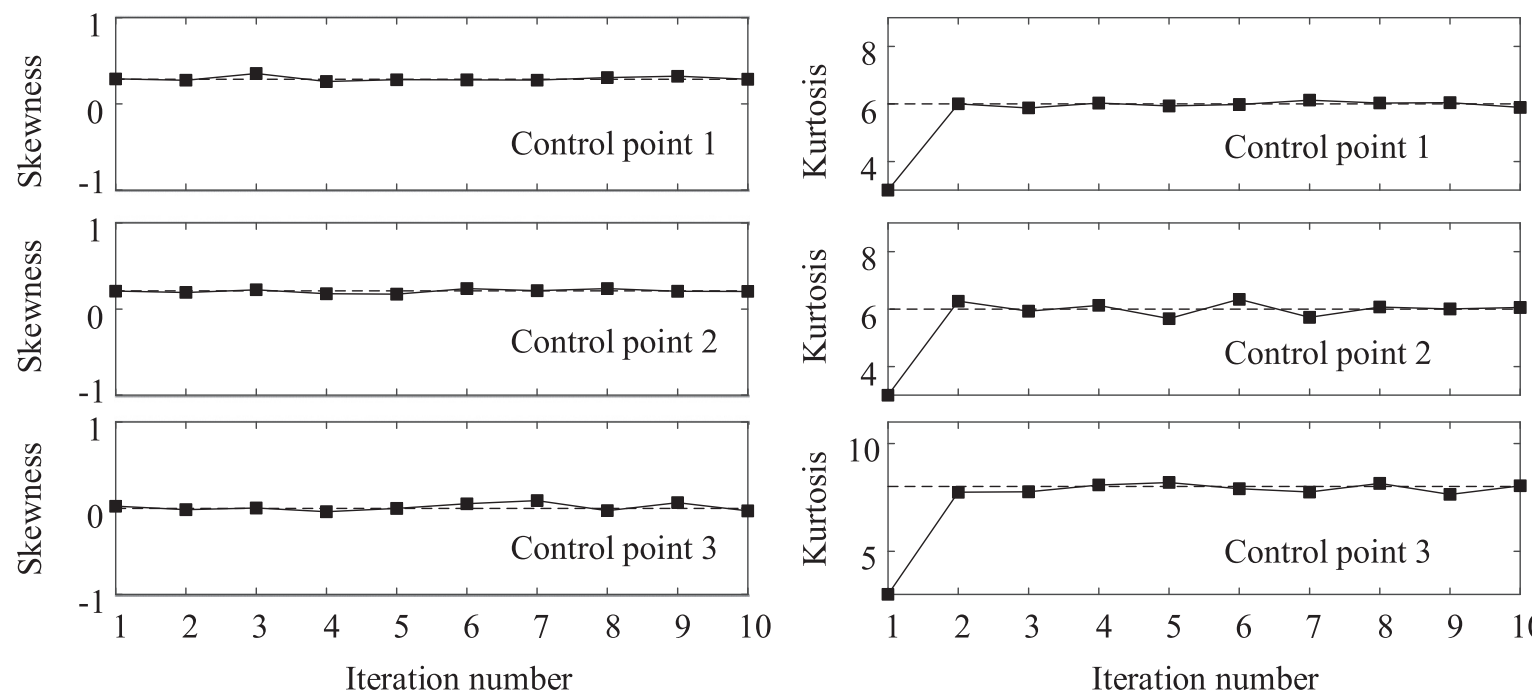

--- Reference skewness $\rightarrow$ Response skewness



Fig. 7. Controlled response skewnesses and kurtoses of three control points.

Fig. 5 shows the uncontrolled response spectra. Auto spectral densities of three control points are not completely within the $3 \mathrm{~dB}$ alarm limit due to a system resonance, and the corresponding cross spectral densities are also not well controlled. The control response spectra are shown in Fig. 6 with the auto spectral densities of all three control points within the $\pm 3 \mathrm{~dB}$ alarm limits. The first ten traces of response skewnesses and kurtoses of three control points are shown in Fig. 7 . The figure clearly shows that the response skewnesses and kurtoses of three control points are stably controlled near their reference 




Control point 2

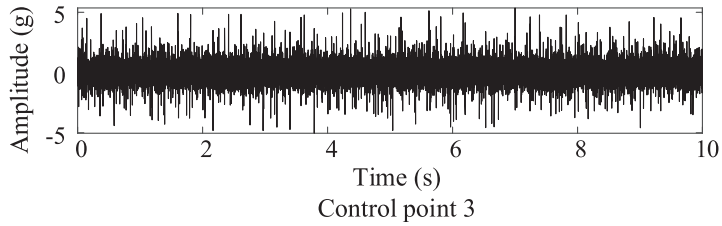

Fig. 8. Controlled response signals segments of three control points.

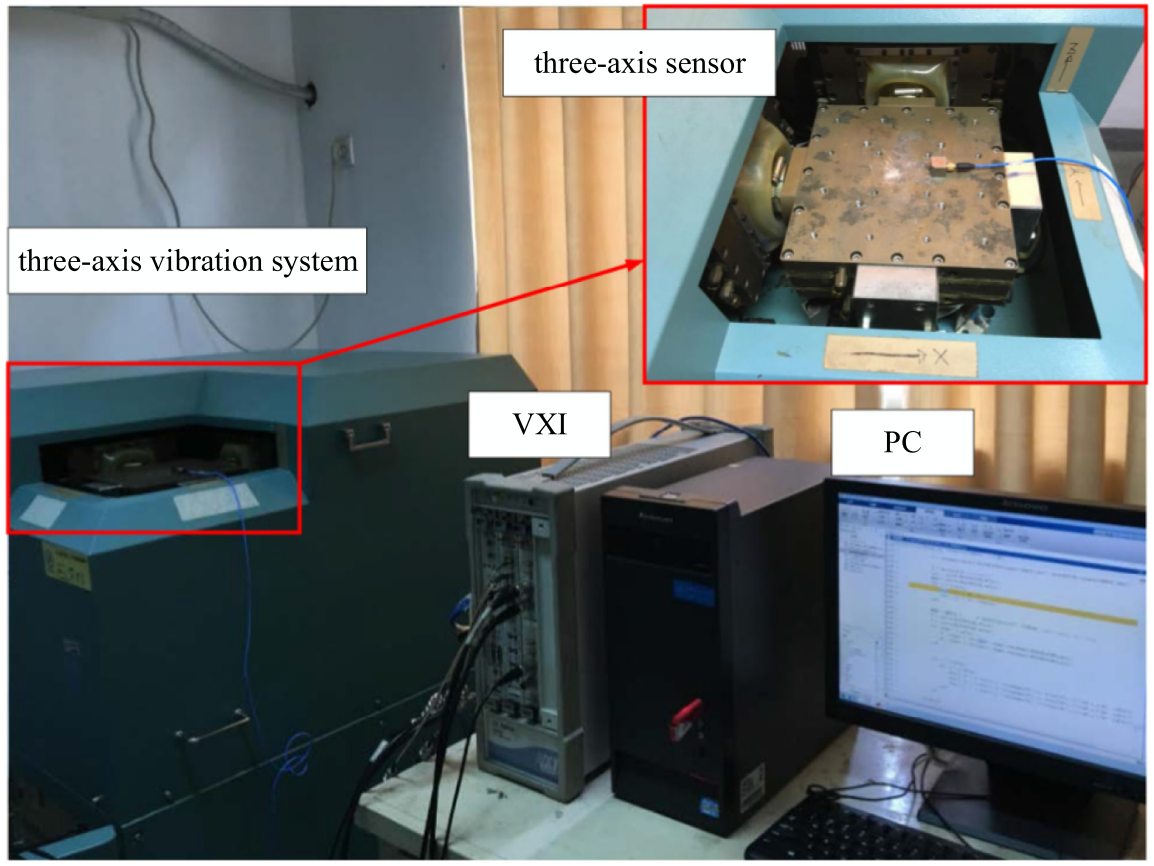

Fig. 9. Triaxial stationary non-Gaussian random vibration test.

values after corrections. Three segments of response signals of three control points exhibit the non-Gaussian nature, as given in Fig. 8.

\section{Experimental results}

To verify the feasibility and validity of the proposed method, a triaxial non-Gaussian random vibration test is carried out. Fig. 9 shows the Agilent VXI hardware which is used to control the three directions x, y and $\mathrm{z}$ of the shaker table. System identification is conducted first to estimate the frequency response function matrix of the test system by running a lowlevel random test and the plots of frequency response functions is shown in Fig. 10. The control frequency band is set from $20 \mathrm{~Hz}$ to $2000 \mathrm{~Hz}$ with 800 spectral lines. The reference spectra of three control directions are set in Tables 4 and 5 . Power spectral densities are numerically specified at the break points only with values at other frequency points are calculated by 

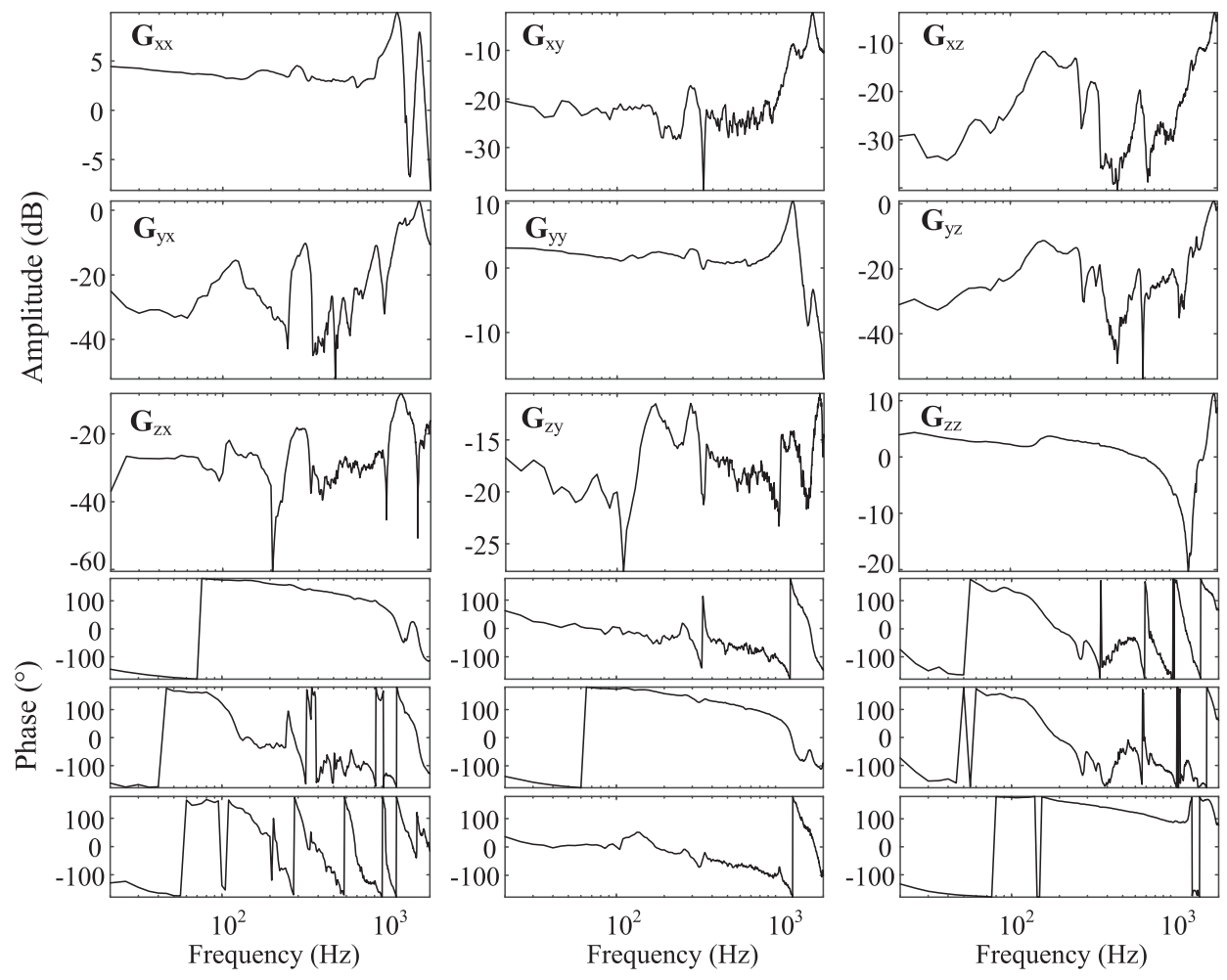

Fig. 10. Plots of frequency response functions of three control directions.

Table 4

Reference auto spectral densities for triaxial vibration test.

\begin{tabular}{llll}
\hline Break point $(\mathrm{Hz})$ & $\mathrm{R}_{\mathrm{xx}}\left(\mathrm{g}^{2} / \mathrm{Hz}\right)$ & $\mathrm{R}_{\mathrm{yy}}\left(\mathrm{g}^{2} / \mathrm{Hz}\right)$ & $\mathrm{R}_{\mathrm{zz}}\left(\mathrm{g}^{2} / \mathrm{Hz}\right)$ \\
\hline 20 & $1 \mathrm{e}-6$ & $1 \mathrm{e}-6$ & $1 \mathrm{e}-6$ \\
100 & $1 \mathrm{e}-5$ & $1 \mathrm{e}-5$ & $1 \mathrm{e}-5$ \\
1000 & $1 \mathrm{e}-5$ & $1 \mathrm{e}-5$ & $1 \mathrm{e}-5$ \\
2000 & $1 \mathrm{e}-6$ & $1 \mathrm{e}-6$ & $1 \mathrm{e}-5$ \\
\hline
\end{tabular}

Table 5

Reference cross spectral densities for triaxial vibration test.

\begin{tabular}{llllll}
\hline Break point $(\mathrm{Hz})$ & Coherence $\gamma_{\mathrm{xy}}^{2}$ & Coherence $\gamma_{\mathrm{xz}}^{2}$ & Coherence $\gamma_{\mathrm{zy}}^{2}$ & Phase $\alpha_{\mathrm{xy}}\left({ }^{\circ}\right)$ & Phase $\alpha_{\mathrm{xz}}\left({ }^{\circ}\right)$ \\
\hline 20 & 0.5 & 0.1 & 0 & 45 & -60 \\
100 & 0.5 & 0.1 & 0 & 45 & -60 \\
1000 & 0.5 & 0.1 & 0 & 45 & -60 \\
2000 & 0.5 & 0.1 & 0 & 45 & -60 \\
\hline
\end{tabular}

Table 6

Reference skewnesses and kurtoses for triaxial vibration test.

\begin{tabular}{lll}
\hline Control direction & Skewness & Kurtosis \\
\hline $\mathrm{x}$ & 0.2 & 6 \\
$\mathrm{y}$ & 0 & 6 \\
$\mathrm{z}$ & -0.2 & 8 \\
\hline
\end{tabular}



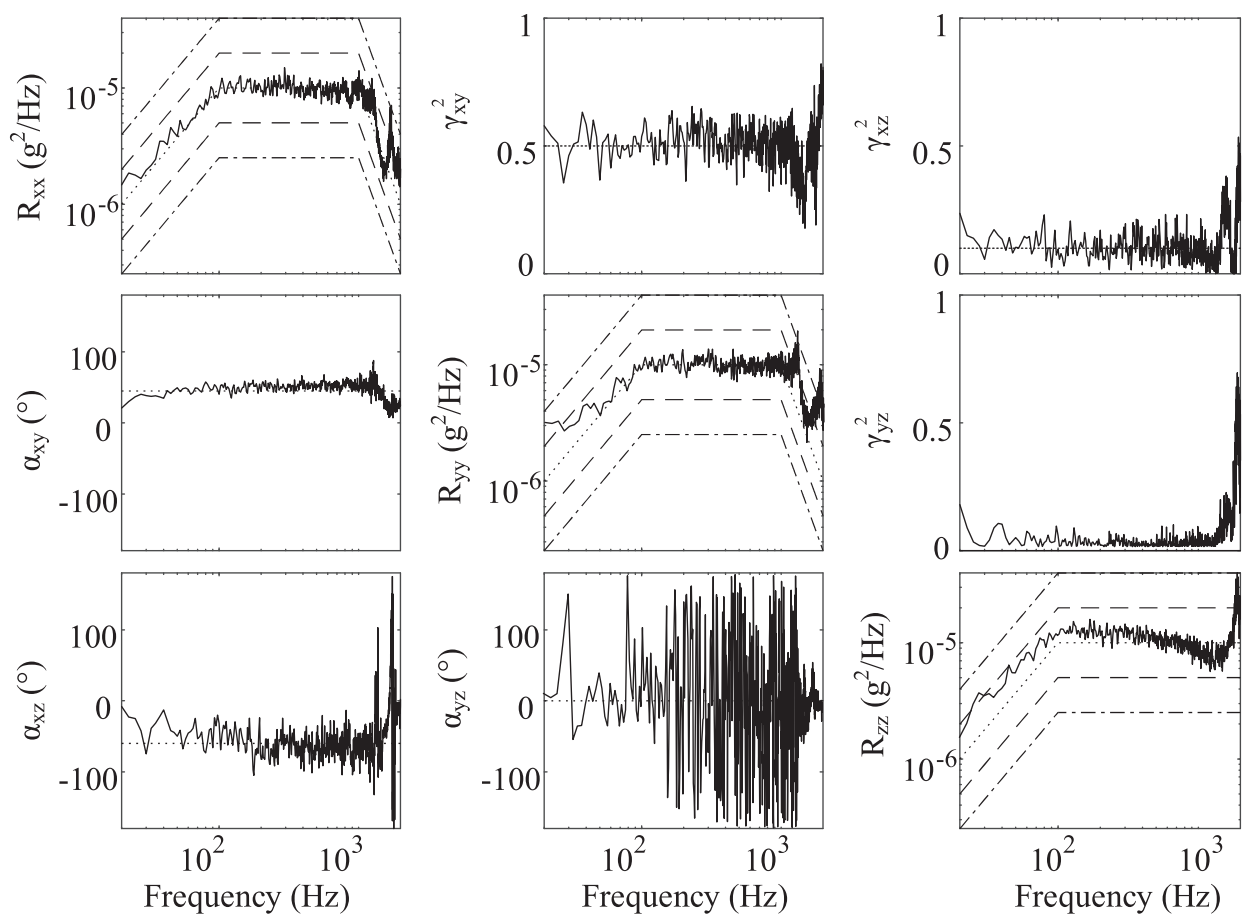

$$
\begin{array}{lr} 
--- \pm 3 \mathrm{~dB} \text { alarm limits } & \text { Reference spectrum } \\
--- \pm 6 \mathrm{~dB} \text { abort limits } & \text { Response spectrum }
\end{array}
$$

Fig. 11. Uncontrolled response spectra of three control directions.
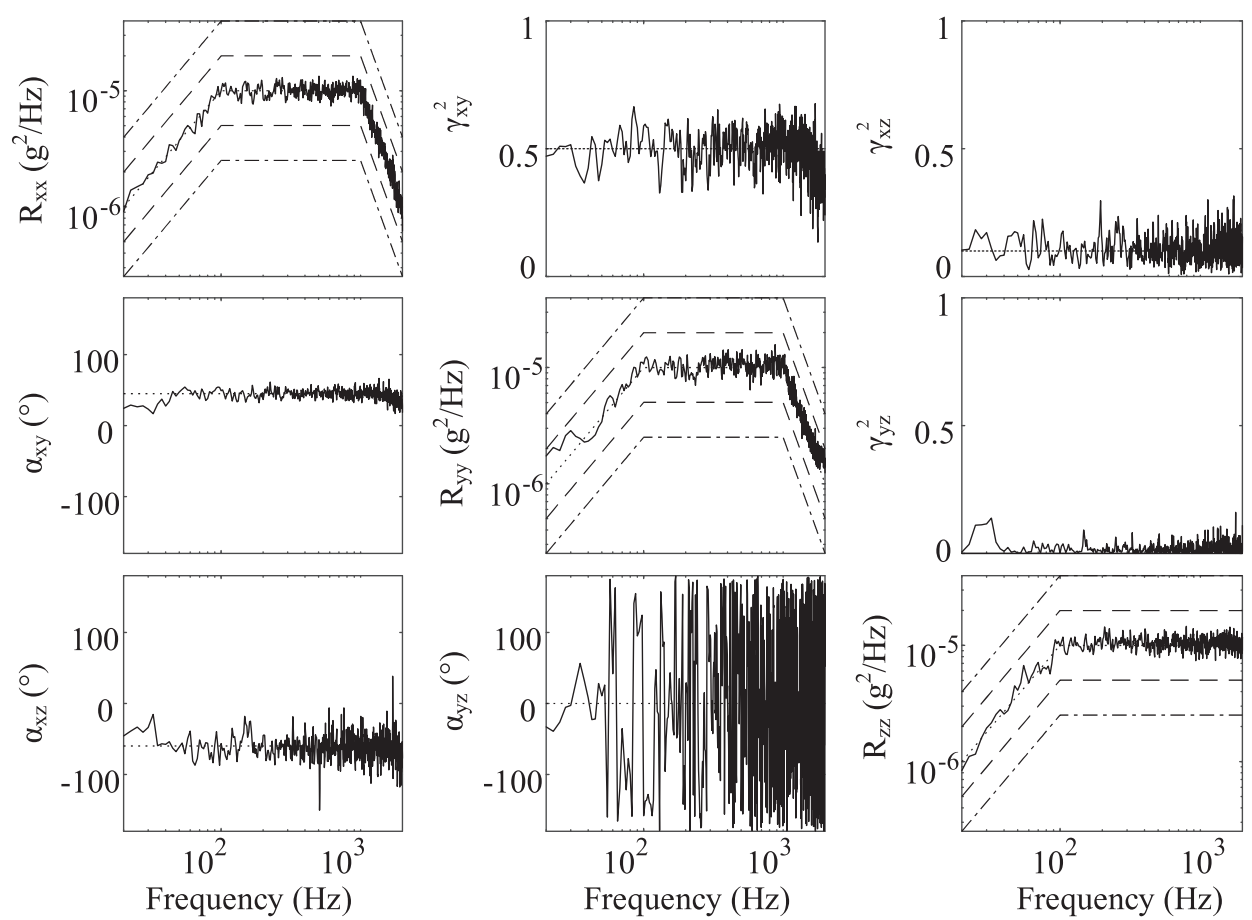

$$
\begin{array}{ll} 
--- \pm 3 \mathrm{~dB} \text { alarm limits } & \text { Reference spectrum } \\
--- \pm 6 \mathrm{~dB} \text { abort limits } & \text { Response spectrum }
\end{array}
$$

Fig. 12. Controlled response spectra of three control directions. 




--- Reference skewness - Response skewness

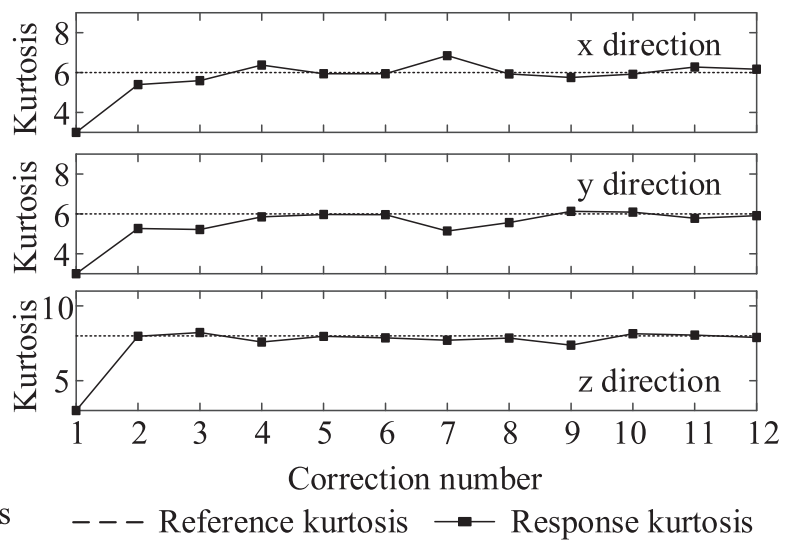

Fig. 13. Controlled response skewnesses and kurtoses of three control directions.

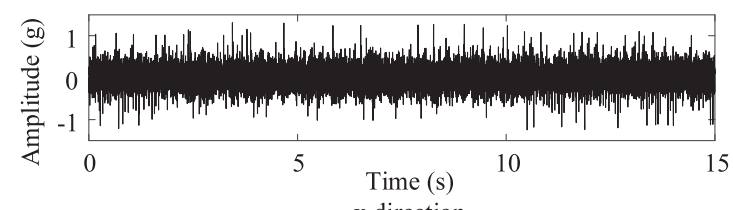

$\mathrm{x}$ direction
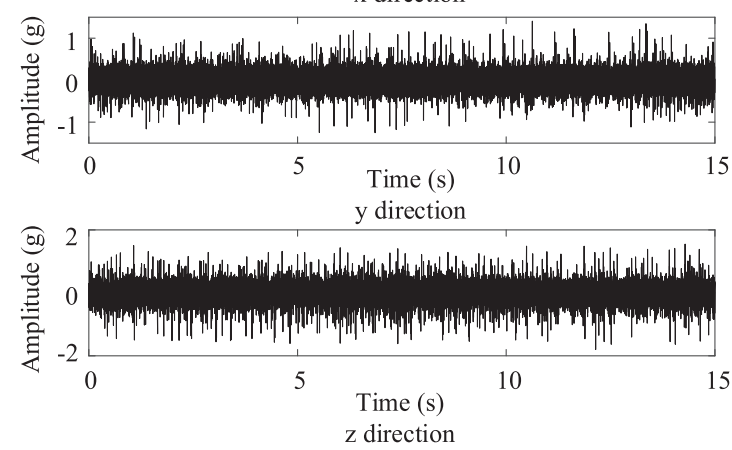

Fig. 14. Controlled response signals segments of three control directions.

linear interpolation on a logarithmic scale. The reference skewnesses and kurtoses of three control directions are set in Table 6.

Fig. 11 shows the uncontrolled response spectra of three control directions. The controlled response spectra after three closed loop corrections are given in Fig. 12 where the auto spectral densities are entirely controlled within the $\pm 3 \mathrm{~dB}$ alarm limits and the cross spectra are also closely controlled. The convergent traces of response skewnesses and kurtoses of three control directions are shown in Fig. 13. The controlled kurtoses are very close to the reference values, and controlled skewnesses are always stable near the reference values. Three time histories of three control directions are given in Fig. 14.

\section{Conclusions}

Random vibration environmental testing is performed to replicate the desired statistical properties of measured dynamic environments by a closed loop control method. Traditional MIMO Gaussian control targets are usually the power spectral densities in the frequency domain. This paper presents a new method for MIMO stationary non-Gaussian random vibration control. The targets for refining the control strategy are the response skewnesses, kurtoses, auto-spectral densities and coherence and phase coefficients.

The generation process of desired drive signals is analyzed and a time domain randomization technique is utilized that makes the generation process computationally efficient. The skewness and kurtosis tuning procedures of each channel are elaborated and the sequential phase modification method are performed to modify the uniformly distributed random phase angles channel by channel in sequence to avoid cross coupling. The problem of definition of reference skewnesses, kurtoses and power spectral densities is addressed and it shows that the setting of reference skewnesses and kurtoses is affected by 
the specified power spectral densities. The results from a numerical case study and experimental data set prove the feasibility and validity of the proposed method.

\section{Acknowledgement}

This work was supported by the China Scholarship Council. (No. 201806830049).

\section{References}

[1] U. Musella, G. D’Elia, A. Carrella, et al, A minimum drives automatic target definition procedure for multi-axis random control testing, Mech. Syst. Sig. Process. 107 (2018) 452-468

[2] S. Cui, H.H. Chen, X.D. He, et al, Control algorithm update for multi-input multi-output random environment test, Mech. Syst. Sig. Process. 111 (2018) 643-662.

[3] M.A. Underwood, T. Keller, R. Ayres, Multi-shaker control: a review of the evolving state-of-the-art, Sound Vibrat. 51 (8) (2017) 8-16.

[4] D.O. Smallwood, Generation of stationary non-Gaussian time histories with a specified cross-spectral density, Shock Vib. 4 (5-6) (1997) 361-377.

[5] P. Böröcz, S.P. Singh, Measurement and analysis of vibration levels in rail transport in central europe, Packag. Technol. Sci. 30 (8) (2017) $361-371$.

[6] M. Gioffre, V. Gusella, M. Grigoriu, Simulation of non-Gaussian field applied to wind pressure fluctuations, Probab. Eng. Mech. 15 (4) (2000) 339-345.

[7] F. Cianetti, M. Palmieri, J. Slavič, et al, The effort of the dynamic simulation on the fatigue damage evaluation of flexible mechanical systems loaded by non-Gaussian and non-stationary loads, Int. J. Fatig. 103 (2017) 60-72.

[8] M. Ernst, E. Habtour, A. Dasgupta, et al, Comparison of electronic component durability under uniaxial and multiaxial random vibrations, J. Electron. Packag. 15 (12) (2015) 957-962.

[9] F. Kihm, N.S. Ferguson, J. Antoni, Fatigue life from kurtosis controlled excitations, Procedia Eng. 133 (2015) 698-713.

[10] R.M. French, R. Handy, H.L. Cooper, A comparison of simultaneous and sequential single-axis durability testing, Exp. Tech. 30 (5) (2010) 32-37.

[11] D. Benasciutti, R. Tovo, Fatigue life assessment in non-Gaussian random loadings, Int. J. Fatig. 28 (7) (2006) $733-746$.

[12] W.E. Whiteman, M.S. Berman, Fatigue failure results for multi-axial versus uniaxial stress screen vibration testing, Shock Vib. 9 (6) (2002) 319-328.

[13] D. O. Smallwood, Multiple shaker random control with cross coupling, in: Proceedings of Combined Environments: Technology Interrelations, 1978, pp. 341-347.

[14] D.O. Smallwood, T.L. Paez, A frequency domain method for the generation of partially coherent normal stationary time domain signals, Shock Vib. 1 (1) (1993) 45-53.

[15] D.O. Smallwood, Generation of partially coherent stationary time histories with non-Gaussian distributions, Office Sci. Tech. Inform. Tech. Rep. (1996).

[16] D.O. Smallwood, Generating non-Gaussian vibration for testing purposes, Sound Vibrat. 39 (10) (2005) $18-24$.

[17] R.H. Zheng, H.H. Chen, X.D. He, Control method for multiple-input multiple-output non-Gaussian random vibration test, Packag. Technol. Sci. 30 (7) (2017) 331-345.

[18] R.H. Zheng, H.H. Chen, X.D. He, Control method for multi-input multi-output non-Gaussian random vibration test with cross spectra consideration, Chin. J. Aeronaut. 30 (6) (2017) 1895-1906.

[19] J. D. Tebbs, N. F. Hunter, Digital control of random vibration tests using a Sigma V computer, in: Proceedings of annual technical meeting and equipment exposition of the Institute of Environmental Sciences, Washington, District of Columbia, USA, 1974. 